\title{
LITERATUR
}

\section{Die EU und der post-sowjetische Raum - politische Konflikte und wissenschaftliche Fragestellungen}

\author{
Sabine Fischer*
}

Die EU-Osterweiterung hat den post-sowjetischen Raum näher an die EU-Außengrenzen heranrücken lassen. Innen- und Außenpolitik der Neuen Unabhängigen Staaten (NUS) sind damit auch für die EU bedeutsamer geworden. Gleichzeitig kollidiert der zunehmende Einfluss der EU mit den ordnungspolitischen Ansprüchen Russlands in der Region, das angesichts seiner Größe und seiner Rolle als Energielieferant mit Abstand der wichtigste, aber auch schwierigste Partner europäischer Politik östlich der EU-Außengrenzen ist. Das ,neue Selbstbewusstsein', mit dem Moskau eine Position als Großmacht in Europa und auf globaler Ebene für sich einfordert, hat das Konfliktpotenzial mit der EU eher noch vergrößert. Diese Entwicklungen sind Gegenstand einer Reihe von 2005 und 2006 erschienen Monographien und Sammelbänden, welche die Beziehungen zwischen der EU und den Staaten des post-sowjetischen Raums aus unterschiedlichen Blickwinkeln analysieren.

\section{Die EU und der Südkaukasus}

Sebastian Mayer wendet sich in seiner Studie der Politik der EU gegenüber Georgien, Armenien und Aserbaidschan zu. Sein Erkenntnisinteresse gilt hauptsächlich außenpolitischen Entscheidungsprozessen innerhalb der EU und dem Verhältnis zwischen Rat und Kommission bei der Formulierung der Südkaukasuspolitik. Nach einer knappen theoretischen Reflexion wendet der Autor sich den Institutionen des außenpolitischen Entscheidungsprozesses in den drei Säulen der EU zu. Er zeichnet ein recht genaues Bild von den in
Sebastian Mayer: Die Europäische Union und der Südkaukasus. Interessen und Institutionen in der Auswärtigen Politikgestaltung, [Nomos Universitätsschriften: Politik, Band 133], Nomos Verlagsgesellschaft: Baden-Baden 2006, ISBN 3-8329-1598-2; 270 Seiten, $44 €$.

Katrin Bastian: Die Europäische Union und Russland. Multilaterale und bilaterale Dimensionen in der europäischen Außenpolitik, VS Verlag für Sozialwissenschaften: Wiesbaden 2006, ISBN 3-5311-5099-5; 300 Seiten, 39,90 €.

Jakob Hedenskog u.a. (Hrsg.): Russia as a Great Power. Dimensions of security under Putin, Routledge: London/New York 2006, ISBN 04153-5996-1; 432 Seiten, $75 £$.

Debra Johnson und Paul Robinson (Hrsg.): Perspectives on EU-Russia Relations, [Europe and the nation state, Band 7], Routledge: London/ New York 2005, ISBN 0-4153-3985-5; 224 Seiten, $70 £$.

Kirsten Westphal (Hrsg.): A Focus on EU-Russian Relations. Towards a close partnership on defined road maps?, [Schriften zur Internationalen Entwicklungs- und Umweltforschung, Band 15], Peter Lang: Frankfurt am Main u.a. 2005, ISBN 3-6315-4257-7, 114 Seiten, 24,80 €.

Sergei Prozorov: Understanding Conflict between Russia and the EU. The Limits of Integration, Palgrave: Basingstoke 2006, ISBN 1-40399689-X, 240 Seiten, $45 £$.

Rolf Peter: Russland im neuen Europa. Nationale Identität und außenpolitische Präferenzen (19922004), [Studien zu Konflikt und Kooperation im Osten, Band 15], LIT-Verlag: Münster u.a. 2006, ISBN 3-8258-9046-5, 344 Seiten, 24,90€.

* Dr. Sabine Fischer, Forschungsgruppe Russland / GUS, Stiftung Wissenschaft und Politik, Berlin. 
der Kommission mit Außenwirtschaftsbeziehungen befassten Organen und ihrem Verhältnis zum Rat und der GASP beziehungsweise zum Europäischen Parlament.

Die empirische Darstellung, die den überwiegenden Teil der Studie einnimmt, beschreibt die Entwicklung der EU-Politik im Südkaukasus seit der Auflösung der Sowjetunion. Zwei Motive werden als besonders dominant hervorgehoben: die Sicherung der Energieversorgung durch wirtschaftliche Aufbauhilfe und Einbindung der drei Staaten in multilaterale Energieverträge (Energiecharta) sowie die Stabilisierung der Region durch Maßnahmen der Demokratieförderung und Konfliktbearbeitung. Mayer weist nach, dass die EU ab Mitte der 1990er Jahre eine immer aktivere Südkaukasuspolitik betrieben habe. Dies liege auch darin begründet, dass der Rat beziehungsweise die Mitgliedstaaten größeres Interesse an der Region entwickelt habe, während in der ersten Hälfte der 1990er Jahre die Kommission über weit gehende Gestaltungsfreiheit verfügt habe. Der im wissenschaftlichen Diskurs verbreiteten Einschätzung, die EU sei in der Region ein „weak player" (S. 246) geblieben, stellt Mayer die These entgegen, Brüssel habe zwar keine klassische Beziehungsmacht gewonnen, jedoch in der Region seit Anfang der 1990er Jahre die Position einer „strukturellen Macht“ aufgebaut. Darunter versteht der Autor die Fähigkeit eines Akteurs, Interaktionen so zu kontrollieren, ,dass die Strukturen, die daraus entstehen, langfristig den Zielen eines Akteurs dienen“" (S. 247).

Es ist das Verdienst von Sebastian Mayer, eine geschlossene Abhandlung über die Südkaukasus-Politik der EU vorgelegt zu haben. Dies ist in monographischer Form noch nicht geleistet worden, was sicherlich nicht zuletzt daran liegt, dass diese Region bislang nicht im Fokus europäischer Politik lag. Gleichzeitig weist die Studie jedoch einige vor allem konzeptionelle Schwächen auf. Die Berufung sowohl auf rationalistische als auch auf konstruktivistische, auf akteurstheoretische wie auf institutionalistische Ansätze lässt den Versuch einer Kon- zeptionalisierung ins Beliebige abgleiten und bietet keinen roten Faden für die folgende empirische Analyse. Hier trägt der Autor viele wertvolle Informationen zusammen, die jedoch bedauerlicherweise nicht systematisch strukturiert werden. Stattdessen folgt in den sechs empirischen Kapiteln auf eine lange Deskription zumeist nur eine zu kurze Analyse, die dann wichtige Aspekte außer Acht lässt. Darüber hinaus findet keine systematische Bearbeitung, Verknüpfung oder - ebenfalls denkbar - ein Vergleich zwischen den beiden zentralen Themenschwerpunkten Energiesicherheit und Konfliktbearbeitung statt, die sich durch die gesamte Studie ziehen. Ein weiterer Schwachpunkt ist das Fehlen jeder regionalen Kontextualisierung. Externe Akteure wie die USA und besonders Russland, die für die Formulierung und Entwicklung der EUPolitik im post-sowjetischen Raum von elementarer Bedeutung sind, werden bestenfalls am Rande erwähnt. Schließlich könnte die These, die EU habe im Südkaukasus ,strukturelle Macht" entwickelt, nur auf der Basis einer Wirkungsanalyse von EU-Politik im Südkaukasus formuliert werden. Diese unterlässt der Autor berechtigterweise, da sie nicht im Fokus seines Erkenntnisinteresses liegt. Eine solche Analyse sowie die Untersuchung der Position der EU in den regionalen Beziehungen würde jedoch mit großer Wahrscheinlichkeit zu dem Ergebnis führen, dass die EU zwar möglicherweise nach „struktureller Macht" strebt, dies jedoch im fragmentierten und von Konflikten gezeichneten Südkaukasus bislang keinesfalls ist.

\section{Russlandpolitik der EU}

Auch Kathrin Bastian nimmt in ihrer Studie ganz explizit eine ,europäische Perspektive“ ein. Sie richtet ihr politikwissenschaftliches Interesse auf das Zusammenspiel der bilateralen und multilateralen Dimensionen europäischer Außenpolitik, ihre Effektivität und damit letztendlich auch auf die Frage, ob ,die Europäische Union [sich] als außenpolitischer beziehungsweise internationaler Akteur charakterisieren" (S. 18) lässt. Damit konzentriert auch sie sich - 
wie Mayer - auf EU-interne Entscheidungsprozesse. Basierend auf einer breiten, äußerst informierten und gut zu lesenden Diskussion der relevanten Theorien europäischer Integration einerseits sowie zur Außenpolitikanalyse andererseits entwickelt Bastian einen ,,integrierten Bezugsrahmen" für ihre eigene Untersuchung (ab S. 64). Sie orientiert sich dabei an sechs Faktoren (Akteure; außenpolitischer Prozess; Themen/Agenda; Instrumente; Kontext; Output), die auf multilateraler sowie nationaler Ebene gemeinsam das außenpolitische System der Europäischen Union bilden (S. 65).

In diesem Koordinatensystem legt die Autorin ihre Analyse an. Die empirischen Kapitel konzentrieren sich auf die bilateralen Beziehungen zwischen Russland und der EU und die deutsche und finnische Russlandpolitik im multilateralen Kontext der EU. Deutschland und Finnland bieten sich als Fallbeispiele an, da beide Staaten eine besondere Rolle für die Osteuropa- beziehungsweise Russlandpolitik der EU spielen. Die empirischen Kapitel folgen einer parallelen Grobgliederung, die die Vergleichbarkeit der beiden Fallstudien gewährleistet. Insgesamt ist die empirische Analyse mit großer Sorgfalt und auf der Basis breiter Literaturrezeption sowie zahlreicher Interviews ausgeführt.

In der Zusammenfassung ihrer reichhaltigen empirischen Ergebnisse kommt Katrin Bastian zu dem Schluss, dass multilaterales Handeln im Rahmen europäischer Außenpolitik gegenüber Russland sowohl für Deutschland als auch Finnland bestimmte Funktionen erfüllt (ab S. 268): Zum einen nutzten beide Staaten die multilaterale Bühne, um ihren Ideen mehr Gewicht und Durchsetzungsfähigkeit zu verschaffen. Dies gilt im Falle deutscher Politik beispielsweise für das Konzept der Vier Räume mit Russland; Finnland gelang es durch geschicktes Agenda-Setting, die Nördliche Dimension zu einem wichtigen Aspekt in den Beziehungen zwischen Russland und der EU zu machen. Außerdem übertrügen beide Staaten konflikthafte Themen wie Kaliningrad und Tschetschenien auf die multilaterale Ebene, um bilateralen Spannungen mit Russland auszuweichen.

Hinsichtlich der Fähigkeit der EU, Russland als Akteur gegenüberzutreten, resümiert die Autorin, dass ,die EU nach außen dann die größte Kohärenz zeigt, wenn die Konzeption und Implementierung der außenpolitischen Instrumente der Kommission überlassen werden“, während - wie im Falle Russlands - das Gegenteil der Fall ist, ,wenn die außenpolitische Konzeption vorwiegend in die Kompetenz der Mitgliedstaaten, also in die GASP fällt, und die Kommission nur oberflächlich beteiligt wird“" (S. 264). Bilaterale Politik der Mitgliedstaaten könne multilaterale Politik dabei gut ergänzen, jedoch auch - vor allem in ihrer Werteorientiertheit - unterlaufen (S. 272).

Bastians Studie leistet einen lange ausstehenden Beitrag zur Debatte über die Beziehungen zwischen der EU und Russland, indem sie theoretisch angeleitet und konzeptionell differenziert die Probleme und Potenziale europäischer Russlandpolitik in überzeugender Weise offen legt. Dies ist ein wichtiger Schritt in Richtung einer umfassenden Analyse dieses für Europa immer bedeutsameren Beziehungsgeflechts. Freilich kann die Autorin eine umfassende Analyse der Russland-EU-Beziehungen nicht leisten, da sie sich berechtigterweise auf die Ebene europäischer Russlandpolitik konzentriert. Wünschenswert wäre gleichwohl die Berücksichtigung exogener Einflüsse gewesen: Sowohl die Außenpolitik Russlands als auch die Transformationsprozesse im Inneren des Landes hatten seit 1991 erhebliche Rückwirkungen auf die Formulierung europäischer Außenpolitik. ${ }^{1}$ Zwar fließen diese Aspekte punk-

1 Einen vorzüglichen und umfassenden Überblick über die inneren und äußeren Dimensionen russischer Außenund Sicherheitspolitik bietet Jakob Hedenskog u.a. (Hrsg.): Russia as a Great Power. Dimensions of security under Putin, London/New York 2005. Hier finden sich Analysen strategischer Debatten in Russland, der Beziehungen zu wichtigen globalen Akteuren wie der NATO und den USA, der russischen Politik im post-sowjetischen Raum sowie Untersuchungen der wirtschaftlichen, militärischen und gesellschaftlichen Ressourcen russischer Sicherheitspolitik. 
tuell in Bastians Darstellungen ein, doch findet keine systematische Einbeziehung statt. Dies wäre jedoch notwendig, um nicht nur den (intern) evolutionären, sondern auch den (interaktiv) explorativen Charakter europäischer AuBen- und Russlandpolitik sichtbar zu machen.

Um die Integration EU-europäischer und russischer Perspektiven mit Blick auf die Beziehungen zwischen Russland und der EU bemühen sich zwei 2005 erschienene Sammelbände: Debra Johnson und Paul Robinson setzen sich mit ihrem Band in einer Zeit sich intensivierender Kooperation, aber auch zunehmender Konflikte das Ziel, eine Analyse der Ambivalenzen in den gegenseitigen Beziehungen vorzulegen. Zu diesem Zweck werden im ersten thematischen Block unterschiedliche Perspektiven (EU, Russland, Deutschland) auf die bilateralen Beziehungen wiedergegeben. Der zweite Teil untersucht einzelne Politikbereiche wie europäische Sicherheit, die Kooperation im Rahmen der Nördlichen Dimension, den russischen Krieg in Tschetschenien und die russische Wirtschaftspolitik hinsichtlich ihrer Konsequenzen für die Beziehungen zwischen Russland und der EU. Der Band endet mit policy-orientierten Fallstudien zu Direktinvestionen in Russland, Energiebeziehungen und technischer Kooperation im Rahmen von TACIS. Die von Kirsten Westphal herausgegebene, weniger umfangreiche Aufsatzsammlung enthält Beiträge über Energiebeziehungen, Wirtschaftsbeziehungen, Russlands Beziehungen zur WTO sowie russische und europäische AntiTerrorismuspolitik. Durch die Einbeziehung EU-europäischer und russischer Autorinnen und Autoren präsentieren beide Bände durchaus unterschiedliche Perspektiven. Da die Beiträge keinem einheitlichen konzeptionellen Rahmen folgen bleiben sie jedoch Sammlungen von interessanten Einzelstudien.

\section{Souveränität und Integration}

Sergei Prozorov legt in seiner Monographie den Schwerpunkt auf die Ebene der Interaktion zwischen Russland und der EU. Ausge- hend von den zunehmenden Spannungen zwischen Russland und der EU will er einen Beitrag zum Verständnis der Konflikte leisten und Wege zur Stabilisierung der Beziehungen aufzeigen. In seiner Ausgangshypothese führt der Autor die Konflikte in den Beziehungen zwischen Russland und der EU auf die „fundamental opposition between sovereign and integrationist paradigms" (S. 2) zurück. Sowohl die Politik der EU als auch Russlands seien angeleitet von wechselnden Kombinationen dieser beide Prinzipien. Souveränität begrenzt für Prozorov den Interaktionsraum der im internationalen System handelnden Akteure auf die internationale Ebene und basiert sie auf dem Prinzip souveräner Gleichheit; grenzüberschreitende und homogenisierende Integration ist aus dieser Perspektive nicht denkbar. Souveränität wird so zum Garanten der Freiheit der (eigenen) politischen Gemeinschaft, die ihre Außenpolitik ebenfalls am Prinzip der Souveränität (anderer politischer Gemeinschaften) ausrichten müsse (S. 83). Ergebnis ist ein pluralistisches Bild der internationalen Beziehungen, in dem keine universale Autorität Legitimität für sich beanspruchen könne. Im Gegensatz dazu definiert Prozorov die Logik der Integration, as an attempt to displace the ontological ideas of sovereignty trough the establishment of [...] structures of interaction and cooperation, which are expected to make obsolete the ontological presupposition of the possibility of conflict in international politics" (S. 93). Dieser Verschmelzungsprozess läuft auf die Herrschaft eines friedfertigen universalen $\mathrm{He}$ gemons zu. Dieser beanspruche jedoch wiederum Souveränität für sich, die so lange mit der Souveränität anderer Akteure kollidiere, wie seine Herrschaft noch nicht universal sei. Damit verbleibe auch das Konzept der Integration innerhalb des Souveränitätsparadigmas und sei mit ihm unauflöslich verwoben (S. 93).

Aus den beiden interdependenten Konzepten der Souveränität und der Integration entwickelt Prozorov eine Vierfeldermatrix, deren Interaktionstypen ihm als Modell für eine in- 
terpretative Analyse von Konflikt und Konvergenz in den Beziehungen zwischen Russland und der EU dienen (ab S. 99): Wo beide Seiten gleichermaßen im Rahmen des Souveränitätsparadigmas handeln, kommt es zu gegenseitiger Abgrenzung gleichwertiger, souveräner Einheiten. Werden bilaterale Beziehungen von der Logik der Integration geprägt entstehen „Gemeinsame Räume“.2 Nimmt eine Seite das Handeln der anderen als souveränitätsorientiert wahr und widerspricht dies der eigenen Handlungslogik, reagiert sie mit Exklusion. Selbst-Exklusion schließlich betreibt eine Seite, wenn sie die Handlungslogik der anderen Seite als integrativ und gleichzeitig gegen die eigene Souveränität gerichtet wahrnimmt.

In den empirischen Teilen seiner Studie weist Prozorov in den Beziehungen zwischen Russland und der EU alle vier Interaktionstypen nach: Das von russischer Seite 2004 geäuBerte Ansinnen, die Visapflicht im Reiseverkehr mit der EU aufzuheben, entspricht der Handlungslogik der Integration. Die abwehrende Haltung der EU sei dem Wunsch nach Wahrung ihrer Souveränität entsprungen und deshalb auf Exklusion Russlands ausgerichtet gewesen. Umgekehrt folgt die EU mit ihrer reformunterstützenden technischen Hilfe in Gestalt von TACIS einer auf (hierarchische) Integration abzielenden Politik, während Russland sich dieser mit Verweis auf seine Souveränität zunehmend verschließt (SelbstExklusion).

Damit spitzt sich die Argumentation Prozorovs fast zwangsläufig auf Abgrenzung als Weg zur Stabilisierung der Russland-EU-Beziehungen zu. Er widerspricht jedoch der Annahme, die Handlungslogik der Souveränität führe automatisch in die Hobbesianische Formel des Krieges aller gegen alle (ab S. 147). Kooperation zum Beispiel im Bereich der Sicherheitspolitik sei im souveränen Paradigma keineswegs ausgeschlossen. Der Autor prägt für diese Form der Kooperation den Begriff der „Interaktion ohne Integration“ (S. 156). Allerdings sei die Voraussetzung dafür die gegenseitige Anerkennung als souveräne Subjekte auf internationaler Ebene. Der „Versuchung der Integration" müsse widerstanden werden ,to create intersubjective common spaces of cooperation that are not confined within the limits inherent in every project of integration" (S. 184).

Prozorov legt eine theoretisch fundierte Studie vor, die auch deshalb besonders interessant ist, weil er seine russische Perspektive gekonnt in westlichen Debatten über Theorien der internationalen Beziehungen verankert. Indem er zwei in diesen Debatten heimische Konzepte, nämlich Integration und Souveränität, als bestimmend sowohl für die Politik der EU als auch Russlands nachweist, bekräftigt er seine Ablehnung kulturalistischer Diskurse, die von der Unvereinbarkeit westlicher und russischer Identitäten ausgehen. Seine Analyse bewegt sich insofern auf der Ebene der bilateralen Beziehungen, als sie die Politiken beider Seiten in der Vierfeldermatrix zwischen Integration und Souveränität verortet und auf diese Weise zu Aussagen über Art und Formen der Interaktion zwischen Russland und der EU kommt. Gleichwohl bleibt an dieser Stelle anzumerken, dass es vor allem die russische Politik beziehungsweise die russischen Diskurse über die Beziehungen zur EU sind, welche der Autor sehr differenziert und in vielen Aspekten innovativ darstellt. Auch über die Praxis der Kooperation zwischen den Partnern erfährt die Leserin interessante Details, die sich so kaum in der meist sehr auf die politischen Beziehungen gerichteten Forschung finden. Die Analyse der EUPolitik und der begleitenden EU-europäischen Diskurse ist wesentlich weniger breit fundiert und wirkt streckenweise schematisch. Schließlich hält das kulturalistische Argument, gegen welches sich der Autor einleitend entschieden abgrenzt, durch die Hintertüre

2 Der Begriff wird in Anlehnung an die Vier Gemeinsamen Räume verwendet, die seit 2005 den Orientierungsrahmen für die Weiterentwicklung der Russland-EU-Beziehungen bilden. 
wieder Einzug: Prozorov lehnt die Integration Russlands oder andere Staaten im Sinne einer Übernahme von Normen und rechtlichen Standards der EU ab und plädiert für Pluralismus in den internationalen Beziehungen, lässt sich jedoch auf keinerlei qualitative Diskussion der konkurrierenden Modelle ein. So drängt sich die Frage auf, warum, wenn nicht aus kulturellen Gründen, eine solche Integration unmöglich sei. Auch wenn der Autor diese offene Flanke seiner Argumentation nicht zu schließen vermag, lädt seine Studie gerade aufgrund ihrer sehr seltenen Verankerung in russischen wie westlichen Diskursen zur Diskussion ein.

\section{Identitätsentwürfe russischer Eliten im Wan- del}

Auch Rolf Peter nimmt eine konstruktivistische Perspektive ein, um die Wandlungsprozesse der russischen Europapolitik in der Jelzin-Ära und unter Präsident Putin zu erklären. Er unternimmt einen „Perspektivwechsel auf die Innenseite der russländischen Außenpolitik" (S. 18) und legt den Schwerpunkt seiner Analyse auf die Rolle von Ideen und nationaler Identität. Nur nach Beantwortung der Frage, $o b$ Russland Europa wolle, und welches Europa Russland wolle, lässt sich, so Peter, beurteilen, „inwieweit westeuropäische und russische Konzepte eines gesamteuropäischen Ordnungsmodells vereinbar sind" (S. 19).

Im konzeptionellen Teil seiner Studie unterwirft der Autor die zentralen Begriffe seiner Analyse, Ideen und nationale Identität, einer kritischen Überprüfung. Der gelegentlichen Blindheit des Konstruktivismus für individuelle Handlungskapazitäten und rationalistisches beziehungsweise strategisches Handeln begegnet er mit dem Konzept der Gesellschaftsumwelt, welche ,die politische Wirkung nationaler Identität als sozialen Kontext innerstaatlicher Präferenzbildungsprozesse analytisch greifbar" (S. 53) machen soll. Auf diese Weise soll die Dichotomisierung zwi- schen Instrumentalisierung oder Internalisierung von Ideen durchbrochen werden.

Auf der Basis seiner konzeptionellen Überlegungen führt Peter eine breit angelegte Analyse der europapolitischen Diskurse der russischen außenpolitischen Elite durch. Über die Methode der induktiven Rekonstruktion (S. 99) ermittelt er vier Identitätsentwürfe: westlich-liberaler Universalismus, russisch-liberaler Nationalismus, großrussischer Patriotismus und konservativ-revolutionärer Expansionismus, die jeweils unterschiedliche Vorstellungen von einem europäischen Ordnungssystem und Russlands Rolle darin beinhalten. Das sehr differenzierte Spektrum reicht dabei von der Integration Russlands bei vollständiger Homogenisierung und Anpassung an das Norm- und Regelwerk der EU bis hin zur ,imperialen“ Integration Europas in ein eurasisches Imperium um einen Kernstaat Russland. Diese sehr unterschiedlichen Identitätsentwürfe stehen während des gesamten Untersuchungszeitraums in einem Ideenwettbewerb. Das einzige einende Element sei die Vorstellung von Russland als eine Großmacht - die jedoch nicht zwangsläufig einen Widerspruch zur Identifikation mit Europa bilden müsse (S. 204).

Im zweiten empirischen Teil der Studie untersucht Peter die Wirkung der Identitätsentwürfe in der russischen Europapolitik zwischen 1992 und 2004. Er überprüft die Entwicklung der Außenpolitik auf parallel dazu verlaufenden diskursiven Wandel beziehungsweise Wandlungen in der Gesellschaftsumwelt der Diskurse. Für überwiegende Teile der Jelzin-Ära macht er dissonante Diskurse aus, welche eine unklare und widersprüchliche Politik begleiteten. Die Vereinheitlichung der russischen Europapolitik nach dem Amtsantritt von Vladimir Putin erklärt der Autor mit der gezielten Schaffung eines Staatsdiskurses. Dieser „nahm zentrale Ideen unterschiedlicher, im öffentlichen Diskurs miteinander konkurrierender Identitätsentwürfe auf, wandelte sie ab und kombinierte sie argumentativ auf eine Art und Weise, dass 
potenzielle Antagonismen neutralisiert oder zumindest abgeschwächt wurden“ (S. 294). Dabei blieb Europa von zentraler Bedeutung, doch wurde der auf Anpassung gerichtete Integrationsbegriff des westlich-liberalen Universalismus abgewandelt: „Integration in Europa" bedeutete nun nicht mehr die an EUKriterien ausgerichtete „Europäisierung“ Russlands, sondern ebenbürtige Partnerschaft zwischen Russland und den westeuropäischen Staaten beziehungsweise internationalen Organisationen in einem „Großen Europa“ (S. 294).

Die Studie von Rolf Peter ist aufgrund ihrer vom Mainstream der westlichen Russlandforschung abweichenden Perspektive und ihrer theoretischen und empirischen Versiertheit ein wertvoller Beitrag zur Debatte über die Rolle Russlands im europäischen Ordnungssystem. Besonders die äußerst besonnenen theoretischen und methodischen Reflexionen sowie die breit recherchierte und überzeugend umgesetzte Diskursanalyse machen das Buch lesenswert. Leider versäumt es der Autor, seine theoretischen Ausführungen in einem Analysemodell zu verdichten, welches zu einer stärkeren Systematisierung der drei wesentlichen Faktoren (Ideen, Institutionen, Akteure) im zweiten Teil der empirischen Darstellung hätte führen können. So bleibt das letzte Kapitel deskriptiv, und erst im Schlussteil wird die analytische Qualität des Gedankenmodells wieder sichtbar. Darüber hinaus bleiben die Identitätsentwürfe in der Darstellung merkwürdig statisch. Die Beobachtung, dass im „,neuen“ Staatsdiskurs Module unterschiedlicher Identitätsentwürfe strategisch kombiniert wurden, ist interessant und sicherlich wichtig. Gleichwohl ist anzunehmen, dass eine solche strategische Handlung nur deshalb möglich war, weil die betroffenen
Identitätsentwürfe sich im Laufe der 1990er Jahre bereits gewandelt und aufeinander zu bewegt hatten. Hier billigt Peter Akteuren (etwas zu stark fokussiert auf die Figur des russischen Präsidenten) mehr strategischen Handlungs- und Gestaltungsspielraum zu, als ihnen zur Verfügung stand. Schließlich werden ähnlich wie bei Bastian - externe Einflüsse auf die untersuchten Prozesse konzeptionell und empirisch weitgehend vernachlässigt. Die Bedeutungszunahme Europas im russischen Diskurs beruhte nicht nur auf der Diskreditierung der USA spätestens zum Ende der 1990er Jahre, sondern ist auch auf die Entwicklung und Stärkung europäischer Außenpolitik sowie das zunehmende regionale $\mathrm{Ge}-$ wicht der EU im Vorfeld der EUOsterweiterung zurückzuführen.

\section{Wissenslücken}

Die hier rezensierten Monographien und Sammelbände bilden eine solide Grundlage für die Beschäftigung mit den Beziehungen zwischen der EU und den post-sowjetischen Staaten, allen voran Russland. Allerdings legen sie auch Wissenslücken frei. So fällt ins Auge, dass Studien über die Genese der EUPolitik gegenüber Russland sich auf die EUinternen Entscheidungsprozesse konzentrieren, welchen sie sich mit rationalistisch-institutionalistischen Ansätzen nähern. Was bislang fehlt ist die diskursanalytische Auseinandersetzung mit der Frage, welches Russlandbild den Handlungen unterschiedlicher Akteure in der EU sowie in den Mitgliedsstaaten zugrunde liegt. ${ }^{3}$

Die ideationalen Grundlagen der russischen Europa- und Westpolitik hingegen sind seit Mitte der 1990er Jahre in mehreren konstruktivistischen Studien aufgearbeitet worden. ${ }^{4}$ Auch die

3 Eine Ausnahme, die sich jedoch nicht auf die EU sondern Westeuropa im Allgemeinen bezieht, bildet Iver B. Neumann: Uses of the other. "The East" in European identity formation, Manchester 1999.

4 Vgl. die Arbeiten von Andrei Tsygankov, z.B.: Russia's Foreign Policy. Change and Continuity in National Identity, Lanham/New York 2006; Whose World Order? Russia's Perceptions of American Ideas after the Cold War, Notre Dame 2004; The Final Triumph of the Pax Americana? Western Intervention in Yugoslavia and Russia's Debate On the Post-Cold War Order, in: Communist and Post-Communist Studies 3/2001, S. 133-156. Vgl. auch Sabine Fischer: Russlands Westpolitik in der Krise, 1992-2000, Frankfurt/New York 2003. 
außenpolitischen Entscheidungsprozesse im russischen Transformationssystem sowie die Rolle unterschiedlicher politischer Interessengruppen waren immer wieder Gegenstand wissenschaftlicher Beschäftigung. ${ }^{5}$ Angesichts der erstarkenden russischer Wirtschaft und der zunehmenden Bedeutung von Energie für die Beziehungen zwischen Russland und der EU wäre jedoch ein stärkerer Fokus auf die spezifische Rolle von Wirtschaftsakteuren besonders im Energiesektor für die Formulierung der russischen EU- beziehungsweise Westpolitik wünschenswert. ${ }^{6}$ Die häufig ins Publizistische tendierenden Debatten über die Frage, ob „der Kreml Gazprom“ oder umgekehrt „Gazprom den Kreml“ instrumentalisiere, könnten so auf eine solidere empirische Grundlage gestellt werden.

Schließlich steht die Beantwortung der Frage aus, wie die Demokratiehilfepolitik der EU und anderer westlicher Akteure sich auf den Verlauf der Transformation in Russland und anderen post-sowjetischen Staaten ausgewirkt hat. Analysen von TACIS-Projekten beschränken sich auf Fragen des Projektmanagements und der Effizienz von Einzelprojekten und stellen bestenfalls Vermutungen über den Zusammenhang zwischen ,Demokratiehilfe " und Transformation an. ${ }^{7}$ Um diese offen zu legen ist intensive empirische Forschung nötig, die sowohl von der Kenntnis EU-interner Entscheidungsprozesse als auch der komplexen Implementationsbedingungen in den Transformationsstaaten der Region getragen sein sollte. ${ }^{8}$ Angesichts der Tatsache, dass die immer kritischere Debatte über externe Demokratisierung bislang kaum empirisch unterfüttert ist, eröffnen sich hier aussichtsreiche Forschungsfelder.

5 Neil Malcolm u.a.: Internal Factors in Russian Foreign Policy, Oxford 1996; Dmitri Trenin und Bobo Lo: The Landscape of Russian Foreign Policy-Making, Carnegie Endowment for International Peace 2005; Bobo Lo: Vladimir Putin and the Evolution of Russian Foreign Policy, London 2003.

6 Eine Ausnahme bilden die jüngsten Arbeiten von Heiko Pleines, z.B. Heiko Pleines: Russian Business Interests and the Enlarged European Union, in: Post-Communist Economies 3/2005, S. 269-287.

7 Vgl. Kevin McCann: EU technical assistance programmes and projects: an assessment of energy sector programmes in Eastern Europe and the former Soviet Union, in: Debra Johnson/ Paul Robinson (Hrsg.): Perspectives on EU-Russia Relations, London / New York 2005, S. 194-213.

8 Eine der wenigen Arbeiten, die beide Perspektiven vereint, ist von Tomila Lankina: Explaining European Union Aid to Russia, in: Post-Soviet Affairs 4/2005, S. 309-334. 